\title{
The System Performance of Half-Duplex RELAY NETWORK UNDER EFFECT OF INTERFERENCE NOISE
}

\author{
Miroslav VOZNAK ${ }^{1,3}$, Hoang Quang Minh TRAN ${ }^{2}$, Tan N. NGUYEN ${ }^{3, *}$ \\ ${ }^{1}$ VSB Technical University of Ostrava, 17. listopadu 15/2172, 70833 Ostrava - Poruba, Czech \\ Republic \\ ${ }^{2}$ Optoelectronics Research Group, Faculty of Electrical and Electronics Engineering, Ton Duc \\ Thang University, Ho Chi Minh City, Vietnam \\ ${ }^{3}$ Wireless Communications Research Group, Faculty of Electrical and Electronics Engineering, \\ Ton Duc Thang University, Ho Chi Minh City, Vietnam
}

*Corresponding Author: Tan N. NGUYEN (email:nguyennhattan@tdt.edu.vn)

(Received: 14-October-2017; accepted: 13-December-2017; published: 31-March-2018)

DOI: http://dx.doi.org/10.25073/jaec.201821.75

\begin{abstract}
In recent years, harvesting energy from radio frequency $(R F)$ signals has drawn significant research interest as a promising solution to solve the energy problem. In this paper, we analyze the effect of the interference noise on the wireless energy harvesting performance of a decode-and-forward (DF) relaying network. In this analysis, the energy and information are transferred from the source to the relay nodes in the delay-limited transmission and Delay-tolerant transmission modes by two methods: i) time switching protocol and ii) power splitting protocol. Firstly, due to the constraint of the wireless energy harvesting at the relay node, the analytical mathematical expressions of the achievable throughput, outage probability and ergodic capacity of both schemes were proposed and demonstrated. After that, the effect of various system parameters on the system performance is rigorously studied with closed-form expressions for system throughput, outage probability, and ergodic capacity. Finally, the analytical results are also demonstrated by MonteCarlo simulation. The results show that the analytical mathematical and simulated results agree with each other.
\end{abstract}

\section{Keywords}

Decode-and-forward (DF), relay network, interference noise, wireless energy harvesting.

\section{Introduction}

Nowadays, the fifth generation $(5 \mathrm{G})$ network technology is the best solution for the near future communication network. However, increase the energy efficiency of wireless communication networks is the critical problem, on which are strongly depended the economic and ecological aspects of $5 \mathrm{G}$ networks. For this target, two solutions are proposed and demonstrated. Firstly, overall energy consumption of future $5 \mathrm{G}$ network shall not exceed 10 percent of the current usage. Secondly, much longer battery life for mobile devices is expected [1, 5]. Significant technological steps would have to be taken shortly for this goal to become a reality. Several candidate solutions have been proposed lately to meet the goals above. Technologies based on radio frequency (RF) energy harvesting (EH) and transfer have recently been gaining momentum. With these 
approaches, future wireless devices would have the capability of harvesting energy from signals emitted either by ambient or dedicated sources [1, 8]. In recent years, harvesting energy from radio frequency $(\mathrm{RF})$ signals has drawn significant research interest as a promising solution to solve the energy problem. This energy collection method, referred to as RF energy harvesting, has clear advantages over other energy harvesting techniques due to its predictable, controllable and stable nature. The research in RF energy harvesting mainly falls into two broad categories: Simultaneous Wireless Information and Power Transfer (SWIPT) and Wireless Powered Communication Network (WPCN).

The working principle of SWIPT is that each information signal also carries energy, which can be harvested by energy-limited devices. However, in reality, it is not possible to simultaneously harvest energy and decode information using the same signal. In WPCN, network devices first harvest energy from the signals transmitted by RF power sources and then utilize this harvested energy for their communication needs. In last decade, there are many researchers focus on improving system performance of WPCN. The leading research studied rate-energy trade-off assuming single-inputsingle-output, single-input-multiple-output, and multiple-input-multiple-output setups. The application of wireless energy harvesting to orthogonal frequency division multiplexing [3, 5, and cognitive radio [6] based systems have also been proposed. Moreover, the energy beamforming through wireless energy harvesting has been analyzed for the multi-antenna wireless broadcasting system in [7, 9. Furthermore, secure transmission in the presence of eavesdropper under wireless energy harvesting constraint has been studied in MISO beamforming systems [10, 12]. From this point of view, the system performance of RF energy harvesting communication network is necessary more and more to analyze and study.

In this paper, the effect of the interference noise on the system performance of a wireless energy harvesting. Decode-And-Forward (DF) relaying network was analyzed. In this analysis, the energy and information are transferred from the source to the relay nodes by two methods: time switching protocol and power splitting protocol. Firstly, due to the constraint of the wireless energy harvesting at the relay node, the analytical mathematical expressions of the achievable throughput, outage probability and ergodic capacity of both schemes were presented and demonstrated. After that, the effect of various system parameters on the system performance is rigorously studied with closed-form expressions for system throughput, outage probability, and ergodic capacity. Finally, the analytical results are also demonstrated by Monte-Carlo simulation. The results show that the analytical mathematical and simulated results agree with each other. The main contributions of this paper are summarized as follows:

1) We propose the time switching and the power splitting protocols in the delay-limited and the delay-tolerant transmission modes to enable wireless energy harvesting and information processing at the energy constrained relay in wireless AF relaying networks.

2) The analytical expressions for the achievable throughput, the outage probability and the ergodic capacity for the delay-limited and the delay-tolerant transmission modes is proposed and demonstrated in connection with the various parameters of the system.

3) The influence of the interference noise on the system performance is presented and convinced in details. The rest of the paper is organized as follows. The system model is presented in detail in section II. Sections III proposes and demonstrates the analytical mathematical description of the throughput, outage probability and ergodic capacity of the time switching and power splitting protocol, respectively. Section IV presents the comparison of the simulation and analytical results from various system parameters. Finally, Section V makes some conclusion of this study.

\section{System model}

In this section, DF relaying cooperative network is presented, where the information is transferred from the source $(\mathrm{S})$ to the destination (D), through an energy constrained inter- 
mediate relay $(\mathrm{R})$. In this model, we assume that no connection between the source and the destination because of elimination transmission information. In this model, an intermediate DF relay is used for the transmission of the information from the source to the destination. In this system, the DF relay harvests energy from the signal of the source at first stage, and then the relay transfer the information to the destination by the harvested energy. For this model, the required power of the data decoding process at the relay is negligible in comparison to the signal transmission energy from the relay to the destination [10, 16]. Moreover, $\mathrm{h}$ and $\mathrm{g}$ are the $S \rightarrow R$ and $R \rightarrow D$ channel gains factor, respectively (Fig. 1). In this paper, the energy harvesting and information processing at the relay node are proposed by the time switching and power splitting protocol at the relay.

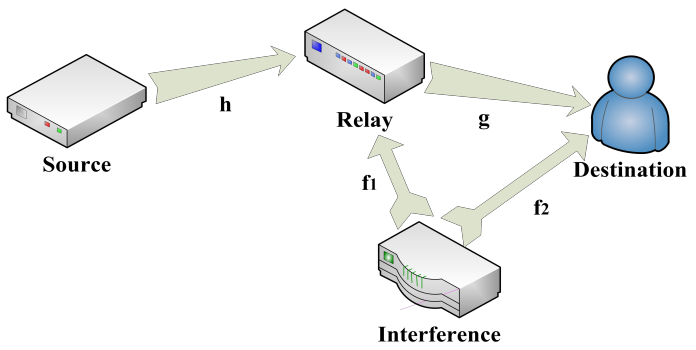

Fig. 1: System model.

For energy harvesting and information processing at the relay by the time switching protocol is presented in Fig. 2. In this scheme, $\mathrm{T}$ is the block time in which the source fully transmits the information data to the destination. Moreover, $\alpha T, \alpha \in(0,1)$ is the time in which the relay harvests energy from the source signal, and $(1-\alpha) T$, is used for information transmission in such a way that half of that, $(1-\alpha) T / 2$, is used for the source to relay information transmission and the remaining half, $(1-\alpha) T / 2$, is used for the relay to destination information transmission. Furthermore, the energy harvesting and information processing at the relay by the power splitting protocol is proposed in Fig. 3. Where $\mathrm{P}$ is the received signal power and $\mathrm{T}$ is the block time (Fig. 3.). Half of the time, $\mathrm{T} / 2$ is used for the source to relay information transmission and the remaining half, $\mathrm{T} / 2$ is used for the relay to destination information transmission. During the first half, the fraction of the received signal power, $\rho P$ is used for energy harvesting and the remaining received power, $(1-\rho) P$ is used for transmitting source information to the relay node, where $\rho \in(0,1)$ [17, 20]. More details of the analytical mathematical model of the achievable throughput and ergodic capacity under the effect of the interference noise (for the time switching and power splitting protocol) is presented in the following sections.

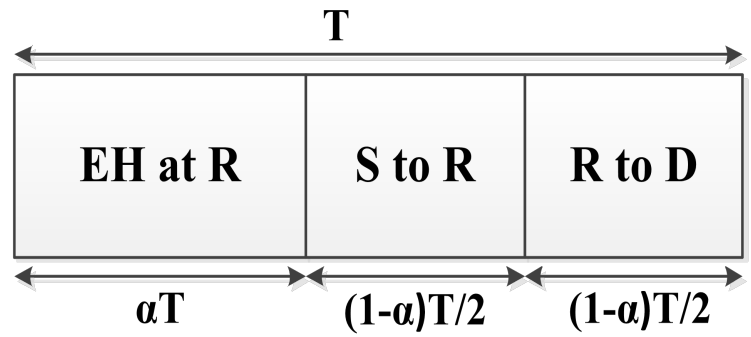

Fig. 2: The energy harvesting and information processing by the time switching protocol.

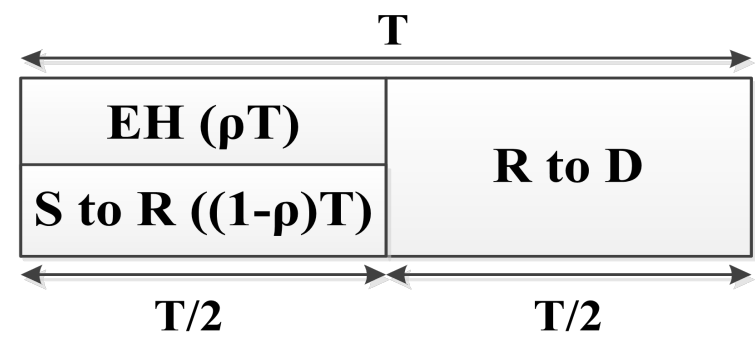

Fig. 3: The energy harvesting and information processing by the power splitting protocol.

\section{The system performance}

\subsection{Delay-limited transmission}

\section{Time Switching Protocol}

$S$ to R Energy Harvesting and Information Transmission The received signal at relay node:

$$
y_{r}=\sqrt{P_{s}} h s(k)+\sqrt{P_{I}} f_{1} i(k)+n_{r}
$$

where $k=1,2, \ldots$ are the symbol index, 

channel,

$h$ is the gain factor from source to relay

$P_{s}$ is the transmitted power from the source,

$P_{I}$ is the interference noise power, node.

$f_{1}$ is the interference function to the relay

$E\left\{\left[s(k)^{2}\right]\right\}=E\left\{\left[i(k)^{2}\right]\right\}=1$,

$E\{$.$\} is the expectation operator.$

The harvested energy at the relay is given by:

$$
E_{h}=\eta \alpha T P_{s}|h|^{2}
$$

In this paper, we assume that the interference power is not large enough for RF energy harvesting at the relay node. So the received power at the relay can be computed:

$$
\begin{aligned}
P_{r} & =\frac{E_{h}}{(1-\alpha) T / 2}=\frac{\eta \alpha T P_{s}|h|^{2}}{(1-\alpha) T / 2} \\
& =\frac{2 \eta \alpha}{1-\alpha} P_{s}|h|^{2}=\kappa P_{s}|h|^{2}
\end{aligned}
$$

In Eq. $\sqrt{22}$ we set $\kappa=\frac{2 \eta \alpha}{1-\alpha}$

$0<\eta<1$ : is the energy conversion efficiency.

The received signal at the destination node:

$$
y_{d}=\sqrt{P_{r}} g r(k)+\sqrt{P_{I}} f_{2} i(k)+n_{d}
$$

Where $g$ is the relay to destination channel gain, tination,

$f_{2}$ is the interference function to the des-

$$
E\left\{\left[r(k)^{2}\right]\right\}=E\left\{\left[i(k)^{2}\right]\right\}=1,
$$

Here $n_{r}, n_{d}$ are the zero mean additive white Gaussian noise (AWGN) with variance $N_{0}$.

The signal to noise ratio for $S \rightarrow R$ links is given by:

$$
\gamma_{r}^{T S}=\frac{E\left\{(\text { signal })^{2}\right\}}{E\left\{(\text { noise })^{2}\right\}}=\frac{P_{s}|h|^{2}}{P_{I}\left|f_{1}\right|^{2}+N_{0}} \approx \frac{P_{s}|h|^{2}}{P_{I}\left|f_{1}\right|^{2}}
$$

\section{R to D Information Transmission}

Similar to S - R links:

$$
\gamma_{d}^{T S}=\frac{P_{r}|g|^{2}}{P_{I}\left|f_{2}\right|^{2}+N_{0}} \approx \frac{P_{r}|g|^{2}}{P_{I}\left|f_{2}\right|^{2}}
$$

Replace Eq.2 into Eq. 5 we have:

$$
\gamma_{d}^{T S}=\frac{P_{r}|g|^{2}}{P_{I}\left|f_{2}\right|^{2}}=\frac{\kappa P_{s}|h|^{2}|g|^{2}}{P_{I}\left|f_{2}\right|^{2}}
$$

\section{Throughput analysis:}

In this section, we need to evaluate the outage probability:

$$
\begin{aligned}
P_{\text {out }}^{T S} & =\operatorname{Pr}\left[\min \left(\gamma_{r}^{T S}, \gamma_{d}^{T S}\right)<\gamma\right] \\
& =\operatorname{Pr}\left[\min \left(\frac{P_{s}|h|^{2}}{P_{I}\left|f_{1}\right|^{2}}, \frac{\kappa P_{s}|h|^{2}|g|^{2}}{P_{I}\left|f_{2}\right|^{2}}\right)<\gamma\right]
\end{aligned}
$$

Where $\gamma=2^{R}-1$ is the SNR threshold and $\mathrm{R}$ is the rate source.

$$
P_{\text {out }}^{T S}=1+b e^{b \mu} \Gamma(-1, b \mu)-b e^{\lambda_{h} b} \Gamma\left(-1, \lambda_{h} b\right)
$$

Where we set

$$
b=\frac{\lambda_{g} P_{I} \gamma}{\lambda_{f_{2}} \kappa P_{s}}, \mu=\frac{\lambda_{f_{1}} P_{s}}{P_{I} \gamma}+\lambda_{h}
$$

Throughput

$$
\tau^{T S}=\left(1-P_{\text {out }}^{T S}\right) \frac{(1-\alpha) R}{2}
$$

\section{Proof:}

From Eq. 7, we have

$$
P_{\text {out }}^{T S}=1-\operatorname{Pr}\left(\frac{P_{s}|h|^{2}}{P_{I}\left|f_{1}\right|^{2}} \geq \gamma, \frac{\kappa P_{s}|h|^{2}|g|^{2}}{P_{I}\left|f_{2}\right|^{2}} \geq \gamma\right)
$$

$$
\begin{aligned}
P_{\text {out }}^{T S}= & 1-\int_{0}^{\infty} \operatorname{Pr}\left(\frac{P_{s} X}{P_{I} Z_{1}} \geq \gamma \mid X=x\right) \\
& \times \operatorname{Pr}\left(\frac{\kappa P_{s} X Y}{P_{I} Z_{2}} \geq \gamma \mid X=x\right) f_{X}(x) d x
\end{aligned}
$$


Where $X=|h|^{2}, Y=|g|^{2}, Z_{1}=\left|f_{1}\right|^{2}, Z_{2}=$ $\left|f_{2}\right|^{2}$

Here, S-R link and R-D link is the Rayleigh fading channel.

After that, we have the probability density function (PDF) of a random variable (RV) $\mathrm{X}, \mathrm{Y}, \mathrm{Z}_{1}, \mathrm{Z}_{2}$ :

$$
f_{\varphi}(x)=\lambda_{\varphi} e^{-\lambda_{\varphi} x}, \text { which } \varphi=\left\{\mathrm{X}, \mathrm{Y}, \mathrm{Z}_{1}, \mathrm{Z}_{2}\right\}
$$

The cumulative density function(CDF) of RV $\varphi$

$$
F_{\varphi}(x)=1-e^{-\lambda_{\varphi} x}
$$

We denote:

$$
\begin{aligned}
I_{1}= & \operatorname{Pr}\left[\left(\frac{P_{s} X}{P_{I} Z_{1}} \geq \gamma \mid X=x\right)\right]=\operatorname{Pr}\left(\frac{P_{s} x}{P_{I} Z_{1}} \geq \gamma\right) \\
& =\operatorname{Pr}\left(Z_{1} \leq \frac{P_{s} x}{P_{I} \gamma}\right)=1-e^{-\frac{\lambda_{f_{1}} P_{s} x}{P_{I} \gamma}}
\end{aligned}
$$

$$
\begin{aligned}
I_{2} & =\operatorname{Pr}\left[\left(\frac{\kappa P_{s} X Y}{P_{I} Z_{2}} \geq \gamma \mid X=x\right)\right]=\operatorname{Pr}\left(Y \geq \frac{P_{I} \gamma Z_{2}}{\kappa P_{s} x}\right) \\
& =\int_{0}^{\infty} f_{Z_{2}}\left(z_{2}\right) d z_{2} \int_{\frac{P_{I} \gamma Z_{2}}{\kappa P_{s} x}}^{\infty} f_{Y}(y) d y
\end{aligned}
$$

$$
\begin{aligned}
I_{2} & =\int_{0}^{\infty} \lambda_{f_{2}} e^{-\lambda_{f_{2}} Z_{2}} e^{-\frac{\lambda_{g} P_{I} \gamma Z_{2}}{\kappa P_{s} x}} d z_{2} \\
& =\frac{\lambda_{f_{2}} \kappa P_{s} x}{\lambda_{f_{2}} \kappa P_{s} x+\lambda_{g} P_{I} \gamma}
\end{aligned}
$$

The outage probability:

$$
\begin{aligned}
& P_{\text {out }}^{T S}= 1-\lambda_{h} \int_{0}^{\infty}\left(I_{1} \times I_{2}\right) e^{-\lambda_{h} x} d x \\
&= 1-\lambda_{h} \int_{0}^{\infty} \frac{\lambda_{f_{2}} \kappa P_{s} x}{\lambda_{f_{2}} \kappa P_{s} x+\lambda_{g} P_{I} \gamma} \\
& \times\left(1-e^{-\frac{\lambda_{f_{1}} P_{s} x}{P_{I} \gamma}}\right) e^{-\lambda_{h} x} d x \\
& P_{\text {out }}^{T S}=1+\lambda_{h} \int_{0}^{\infty} \frac{\lambda_{f_{2}} \kappa P_{s} x}{\lambda_{f_{2}} \kappa P_{s} x+\lambda_{g} P_{I} \gamma} e^{-\frac{\lambda_{f_{1}} P_{s} x}{P_{I} \gamma}} e^{-\lambda_{h} x} d x \\
&-\lambda_{h} \int_{0}^{\infty} \frac{\lambda_{f_{2}} \kappa P_{s} x}{\lambda_{f_{2}} \kappa P_{s} x+\lambda_{g} P_{I} \gamma} e^{-\lambda_{h} x} d x
\end{aligned}
$$

$$
\begin{aligned}
P_{\text {out }}^{T S}= & +\lambda_{h} \int_{0}^{\infty} \frac{x}{x+\frac{\lambda_{g} P_{I} \gamma}{\lambda_{f_{2}} P_{s}}} e^{-\frac{\lambda_{f_{1}} P_{s} x}{P_{I} \gamma}-\lambda_{h} x} d x \\
& -\lambda_{h} \int_{0}^{\infty} \frac{x}{x+\frac{\lambda_{g} P_{I} \gamma}{\lambda_{f_{2}} \kappa P_{s}}} e^{-\lambda_{h} x} d x
\end{aligned}
$$

Where $\lambda_{h}, \lambda_{g}, \lambda_{f_{1}}, \lambda_{f_{2}}$ are the mean values of the exponential random variable $|h|^{2},|g|^{2},\left|f_{1}\right|^{2},\left|f_{2}\right|^{2}$, respectively.

Using Eq [3.383,10] of Table of Integral [21, we have:

$$
P_{\text {out }}^{T S}=1+b e^{b \mu} \Gamma(-1, b \mu)-b e^{\lambda_{h} b} \Gamma\left(-1, \lambda_{h} b\right)
$$

This is the end of the proof.

\section{Power splitting Protocol}

The received signal at relay node:

$$
y_{r}=\sqrt{(1-\rho) P_{s}} h s(k)+\sqrt{P_{I}} f_{1} i(k)+n_{r}
$$

Similar to time switching protocol, the received power at the relay can be computed:

$$
P_{r}=\frac{E_{h}}{T / 2}=\frac{\eta \rho T / 2 P_{s}|h|^{2}}{T / 2}=\eta \rho P_{s}|h|^{2}
$$

The received signal at the destination node:

$$
\begin{gathered}
y_{d}=\sqrt{P_{r}} g r(k)+\sqrt{P_{I}} f_{2} i(k)+n_{d} \\
\gamma_{r}^{P S}=\frac{P_{s}|h|^{2}(1-\rho)}{P_{I}\left|f_{1}\right|^{2}+N_{0}} \approx \frac{P_{s}|h|^{2}(1-\rho)}{P_{I}\left|f_{1}\right|^{2}} \\
\gamma_{d}^{P S}=\frac{P_{r}|g|^{2}}{P_{I}\left|f_{2}\right|^{2}+N_{0}} \approx \frac{P_{r}|g|^{2}}{P_{I}\left|f_{2}\right|^{2}}=\frac{\eta \rho P_{s}|h|^{2}|g|^{2}}{P_{I}\left|f_{2}\right|^{2}}
\end{gathered}
$$

\section{Throughput analysis:}

Similar to Time Switching Protocol, we have the outage probability of system:

$$
\begin{aligned}
P_{\text {out }}^{P S} & =\operatorname{Pr}\left[\min \left(\gamma_{r}^{P S}, \gamma_{d}^{P S}\right)<\gamma\right] \\
& =\operatorname{Pr}\left[\min \left(\frac{P_{s}|h|^{2}(1-\rho)}{P_{I}\left|f_{1}\right|^{2}}, \frac{\eta \rho P_{s}|h|^{2}|g|^{2}}{P_{I}\left|f_{2}\right|^{2}}\right)<\gamma\right] \\
P_{\text {out }}^{P S} & =1+c e^{c \nu} \Gamma(-1, c \nu)-c e^{\lambda_{h} c} \Gamma\left(-1, \lambda_{h} c\right)
\end{aligned}
$$


Where $c=\frac{\lambda_{g} P_{I} \gamma}{\lambda_{f_{2}} \eta \rho P_{s}}, \nu=\frac{\lambda_{f_{1}} P_{s}(1-\rho)}{P_{I} \gamma}+\lambda_{h}$

$\Gamma(\bullet)$ is the gamma function.

Finally, we have the throughput of the system:

$$
\tau^{P S}=\left(1-P_{\text {out }}^{P S}\right) \frac{R}{2}
$$

\subsection{Delay-Tolerant transmission}

\section{Time Switching Protocol}

\section{Throughput analysis:}

In this section, we need to evaluate the ergodic capacity from the source to relay $C_{r}^{T S}$, and for relay to destination link $C_{d}^{T S}$. We use the received signal SNR in (5), (6), respectively. After that $C_{r}^{T S}$ and $C_{d}^{T S}$ are given by the following equations:

$$
\begin{aligned}
& C_{r}^{T S}=\mathrm{E}_{|h|^{2},\left|f_{1}\right|^{2}}\left\{\log _{2}\left(1+\gamma_{r}^{T S}\right)\right\} \\
& C_{d}^{T S}=\mathrm{E}_{|h|^{2},\left|f_{1}\right|^{2},\left|f_{2}\right|^{2}}\left\{\log _{2}\left(1+\gamma_{d}^{T S}\right)\right\} \\
& C_{r}^{T S}=\frac{\ln (\xi)}{\ln 2(\xi-1)}=\frac{\log _{2}(\xi)}{\xi-1}
\end{aligned}
$$

Where we set

$$
\xi=\frac{\lambda_{h} P_{I}}{\lambda_{f_{1}} P_{s}}
$$

The throughput:

$$
\begin{aligned}
C_{d}^{T S}= & \frac{1}{\ln 2} \int_{0}^{\infty} \frac{1}{1+\gamma} \sum_{n=0}^{\infty} \frac{(-1)^{n}\left(\lambda_{f_{2}}\right)^{n+1}}{n !} 4^{n+1} \\
& \times \psi^{-2-2 n} \Gamma(2+n) \Gamma(1+n) d \gamma
\end{aligned}
$$

Where

$$
\psi=\sqrt{\frac{4 \lambda_{h} \lambda_{g} \gamma P_{I}}{\kappa P_{s}}}
$$

Here we set: $C^{T S}=\min \left(C_{r}^{T S}, C_{d}^{T S}\right)$

Proof:

$$
\begin{gathered}
C_{r}^{T S}=\frac{1}{\ln 2} \int_{0}^{\infty} \frac{1-F_{\gamma_{r}^{T S}}(\gamma)}{1+\gamma} d \gamma \\
F_{\gamma_{r}^{T S}}(\gamma)=\operatorname{Pr}\left(\gamma_{r}^{T S}<\gamma\right)=\operatorname{Pr}\left(\frac{P_{s}|h|^{2}}{P_{I}\left|f_{1}\right|^{2}}<\gamma\right) \\
=\operatorname{Pr}\left(\frac{P_{s} X}{P_{I} Y}<\gamma\right)=\operatorname{Pr}\left(X<\frac{\gamma P_{I} Y}{P_{s}}\right)
\end{gathered}
$$

We $\operatorname{set} X=|h|^{2}, Y=\left|f_{1}\right|^{2}$

$$
\begin{aligned}
& F_{\gamma_{r}^{T} S}(\gamma)= \int_{0}^{\infty} f_{Y} d Y \int_{0}^{\frac{\gamma P_{I} Y}{P_{s}}} f_{X} d X \\
&= \int_{0}^{\infty} f_{y} d y\left[1-\exp \left(-\frac{\lambda_{h} \gamma P_{I} Y}{P_{s}}\right)\right] \\
&=1-\int_{0}^{\infty} \lambda_{f_{1}} \exp \left(-\lambda_{f_{1}} Y\right) \\
& \times \exp \left(-\frac{\lambda_{h} \gamma P_{I} Y}{P_{s}}\right) d Y
\end{aligned}
$$

$$
F_{\gamma_{r}^{T S}}(\gamma)=1-\frac{1}{1+\xi \gamma}
$$

Replace into equation (30), we have:

$$
C_{r}^{T S}=\frac{1}{\ln 2} \int_{0}^{\infty} \frac{1}{(1+\xi \gamma)(1+\gamma)} d \gamma=\frac{\log _{2}(\xi)}{\ln 2(\xi-1)}
$$

$$
C_{d}^{T S}=\frac{1}{\ln 2} \int_{0}^{\infty} \frac{1-F_{\gamma_{d}^{T S}}(\gamma)}{1+\gamma} d \gamma
$$

We denote $\vartheta=|h|^{2}|g|^{2}, Z=\left|f_{2}\right|^{2}$

$$
\begin{aligned}
F_{\gamma_{d}^{T S}}(\gamma)= & \operatorname{Pr}\left(\gamma_{d}^{T S}<\gamma\right)=\operatorname{Pr}\left(\frac{\kappa P_{s}|h|^{2}|g|^{2}}{P_{I}\left|f_{2}\right|^{2}}<\gamma\right) \\
& =\operatorname{Pr}\left(\frac{\kappa P_{s} \vartheta}{P_{I} Z}<\gamma\right)=\operatorname{Pr}\left(\vartheta<\frac{\gamma P_{I} Z}{\kappa P_{s}}\right)
\end{aligned}
$$

$$
\begin{aligned}
& F_{\gamma_{d}^{T} S}(\gamma)=\int_{0}^{\infty} f_{Z} \operatorname{Pr}\left(\vartheta<\frac{\gamma P_{I} Z}{\kappa P_{s}}\right) d Z \\
& =\int_{0}^{\infty} f_{Z} d Z \\
& \cdot\left[1-\int_{0}^{\infty} \lambda_{g} \exp \left(-\lambda_{g} y\right) \exp \left(-\frac{\lambda_{h} \gamma P_{I} Z}{\kappa P_{s} y}\right) d y\right]
\end{aligned}
$$

Using the equation $(3.324,1)$ in [21, we have:

$$
\begin{aligned}
F_{\gamma_{d}^{T S}}(\gamma)= & -\int_{0}^{\infty} \lambda_{f_{2}} e^{-\lambda_{f_{2}} Z} \sqrt{\frac{4 \lambda_{h} \lambda_{g} \gamma P_{I} Z}{\kappa P_{s}}} \\
& K_{1}\left(\sqrt{\frac{4 \lambda_{h} \lambda_{g} \gamma P_{I} Z}{\kappa P_{s}}}\right) d Z
\end{aligned}
$$


We set $t=\sqrt{Z}$

$F_{\gamma_{d}^{T S}}(\gamma)=1-2 \int_{0}^{\infty} \begin{array}{ll}\lambda_{f_{2}} t^{2} e^{-\lambda_{f_{2}} t^{2}} \sqrt{\frac{4 \lambda_{h} \lambda_{g} \gamma P_{I}}{\kappa P_{s}}} \\ K_{1}\left(t \sqrt{\frac{4 \lambda_{h} \lambda_{g} \gamma P_{I}}{\kappa P_{s}}}\right)\end{array}$

Apply Taylor series of

$e^{-\lambda_{f_{2}} t^{2}}=\sum_{n=0}^{\infty} \frac{\left(-\lambda_{f_{2}} t^{2}\right)^{n}}{n !}=\sum_{n=0}^{\infty} \frac{\left(-\lambda_{f_{2}}\right)^{n}}{n !} t^{2 n}$

We have:

$$
\begin{aligned}
F_{\gamma_{d}^{T S}}(\gamma)=1-2 \psi \sum_{n=0}^{\infty} & \frac{(-1)^{n}\left(\lambda_{f_{2}}\right)^{n+1}}{n !} \\
& \int_{0}^{\infty} t^{2+2 n} K_{1}(\psi t) d t
\end{aligned}
$$

We apply eq[6.561,16] of Table of Integral [21, we have:

$$
\begin{aligned}
F_{\gamma_{d}^{T S}}(\gamma)= & -\sum_{n=0}^{\infty} \frac{(-1)^{n}\left(\lambda_{f_{2}}\right)^{n+1}}{n !} 4^{n+1} \\
& \times \psi^{-2-2 n} \Gamma(2+n) \Gamma(1+n)
\end{aligned}
$$

$\Gamma(\bullet)$ is the gamma function.

It is the end of the proof.

The throughput of the system:

$$
\tau^{T S}=\frac{(1-\alpha) C^{T S}}{2}
$$

\section{Power splitting Protocol}

$$
\begin{gathered}
C_{r}^{P S}=\frac{\log _{2}(\chi)}{\ln 2(\chi-1)} \\
\chi=\frac{\lambda_{h} P_{I}}{\lambda_{f_{1}} P_{s}(1-\rho)} \\
C_{d}^{P S}=\frac{1}{\ln 2} \int_{0}^{\infty} \frac{1}{1+\gamma} \sum_{n=0}^{\infty} \frac{(-1)^{n}\left(\lambda_{f_{2}}\right)^{n+1}}{n !} 4^{n+1} \\
\times \theta^{-2-2 n} \Gamma(2+n) \Gamma(1+n) d \gamma
\end{gathered}
$$

Where $\theta=\sqrt{\frac{4 \lambda_{h} \lambda_{g} \gamma P_{I}}{\eta \rho P_{s}}}$

$$
C^{P S}=\min \left(C_{r}^{P S}, C_{d}^{P S}\right)
$$

The throughput of the system:

$$
\tau^{P S}=\frac{C^{P S}}{2}
$$

We do not need proof for second protocol because of similar proof as the first protocol.

\section{Results and Discussion}

In this segment, the throughput performance and the ergodic capacity of an energy harvesting DF relaying network under the effect of the interference noise are analyzed in details. The system performance is analyzed in connection with the $\eta, \lambda, \rho, P_{s}$ and $P_{I}$ under the interference noise effect. We consider a network with one source, one relay, and one destination, where source-relay and relay-destination distances are both normalized to unit value. Other simulation parameters are listed in Tab. 1

Tab. 1: Simulation parameters.

\begin{tabular}{|c|c|c|}
\hline Symbol & Name & Values \\
\hline$\eta$ & Energy harvesting efficiency & 0.6 \\
\hline$\lambda_{h}$ & Mean of $|h|^{2}$ & 0.5 \\
\hline$\lambda_{g}$ & Mean of $|g|^{2}$ & 0.5 \\
\hline$\lambda_{f_{1}}$ & Mean of $\left|f_{1}\right|^{2}$ & 1 \\
\hline$\lambda_{f_{2}}$ & Mean of $\left|f_{2}\right|^{2}$ & 1 \\
\hline Ps & The transmit power at source & $0-30 \mathrm{~dB}$ \\
\hline
\end{tabular}

Fig. 4. presents the analytical mathematical and simulation results of throughput in the varied value of $\eta$ with the delay-limited transmission (a) and the delay-tolerant transmission (b). In this simulation, the power $P_{s}$ and $P_{I}$ are set at $30 \mathrm{~dB}$ and $10 \mathrm{~dB}$, respectively. From the Fig. 4 the achievable throughput of the delay-limited and the delay-tolerant transmission modes are increased significantly when the $\eta$ varied from 0 to 1 . Furthermore, the analytical mathematical and simulation results of throughput, concerning $\alpha$ and $\rho$, for the time switching and the power splitting protocols in the delay-limited transmission mode is demonstrated in Fig. 5(a). 
and in the delay-tolerant transmission mode in Fig. 5(b) respectively. In the time switching protocol, the achievable throughput had an optimal value with the value $\alpha$ around 0.2 , and in the power splitting protocol with the value $\rho$ around 0.6. In both schemes, the throughput increased while $\alpha$ and $\rho$ increased to the optimal values, and then decreased.

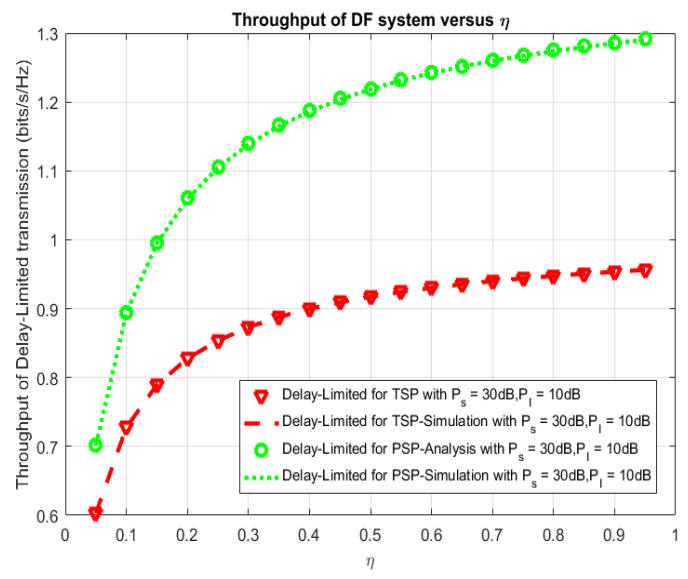

(a)

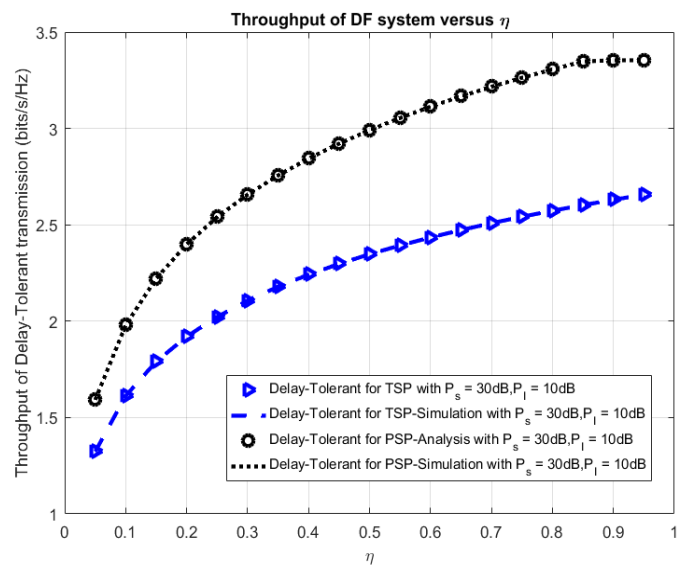

(b)

Fig. 4: Simulation and analytical throughput versus $\eta$ at the destination node.

For more detail analysis, Fig. 6(a)plots the dependent of the throughput on the interference power $P_{I}$ in the delay-limited transmission mode and for the delay-tolerant transmission mode in the Fig. 6(b) for the time switching and the power splitting protocols, respectively.

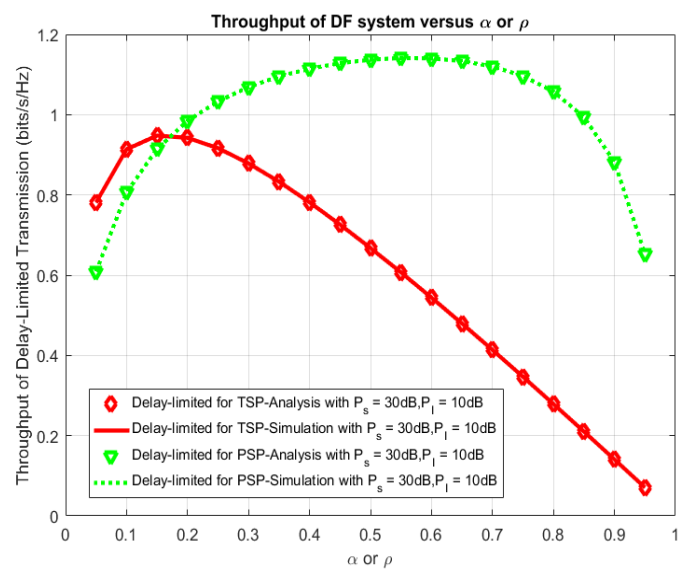

(a)

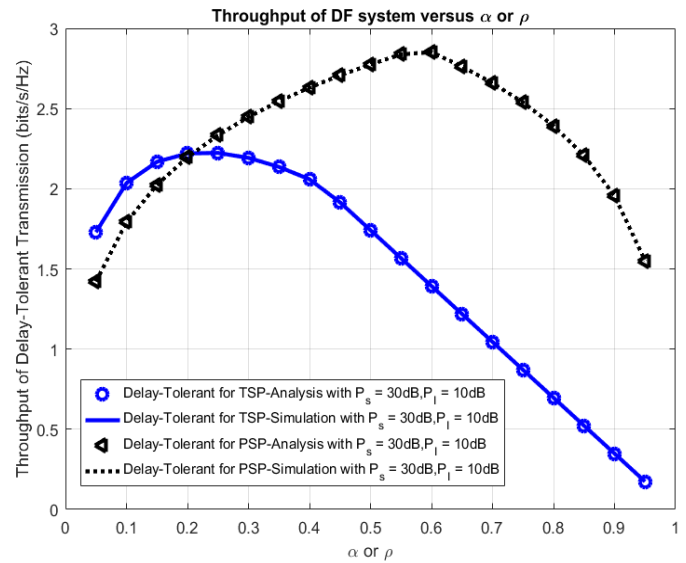

(b)

Fig. 5: Simulation and analytical throughput at the destination concerning $\alpha$ for the time switching and $\rho$ for power splitting protocols.

Both Figs show that the achievable throughput decreased while the $P_{I}$ increased from -10 $\mathrm{dB}$ to $10 \mathrm{~dB}$. In the same way, the Fig. 7 indicates the influence of the source power on the throughput and the outage probability in the delay-limited transmission mode. Then, the influence of the source power on the throughput and the ergodic capacity in the delay-tolerant transmission mode is presented in the Fig. 8. All these results are considered in the time switching and the power splitting protocols. Furthermore, the comparison between the throughput in the delay-limited and the delay-tolerant transmission modes for the time switching and the power 
splitting protocols is proposed in Fig. 9. In this analysis, the analytical mathematical throughput results are calculated by the analytical expressions of throughput and the simulation results based on the equations (in the second section). The research results indicated that the analytical mathematical result and the simulation result based on Monter Carlo analysis are totally matched each other.

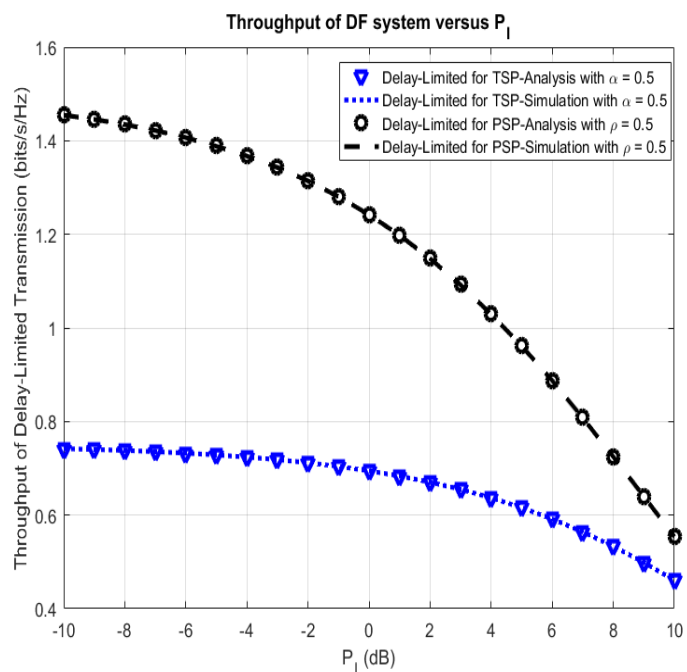

(a)

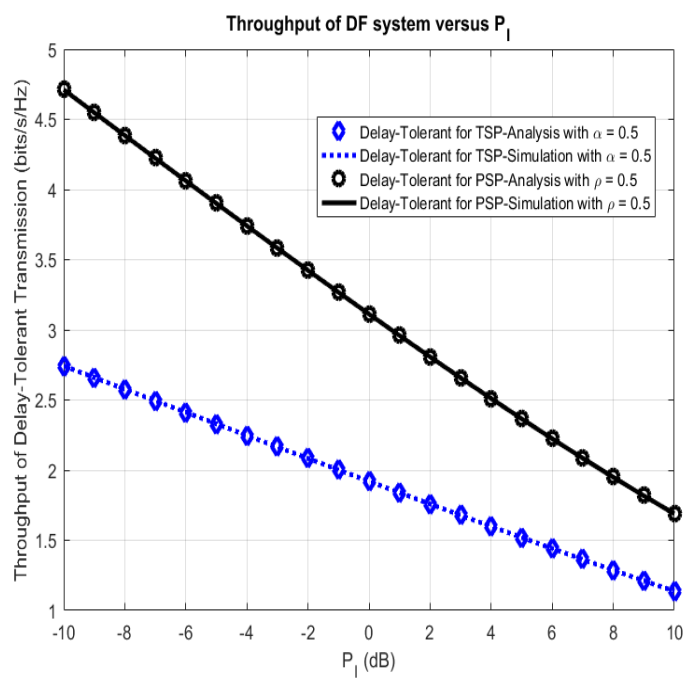

(b)

Fig. 6: Throughput of DF system versus $P_{I}$

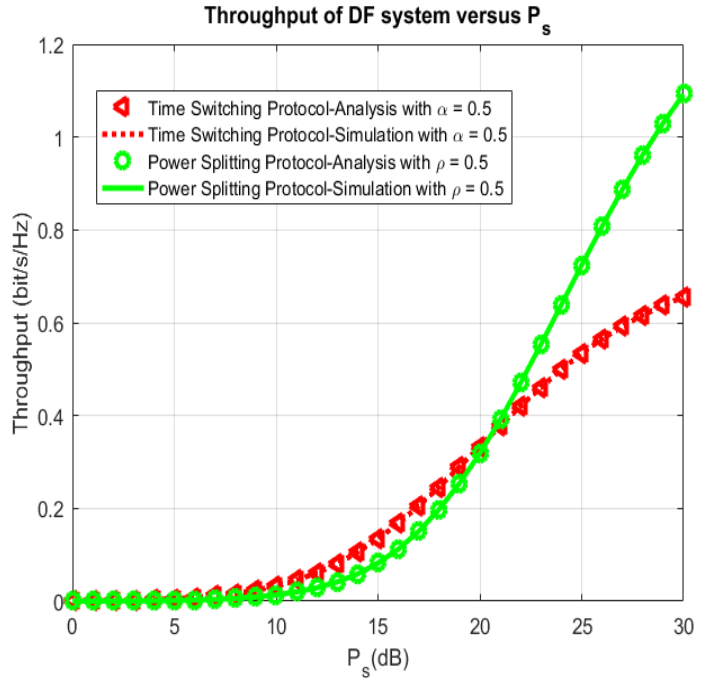

(a) Throughput versus $P_{s}$

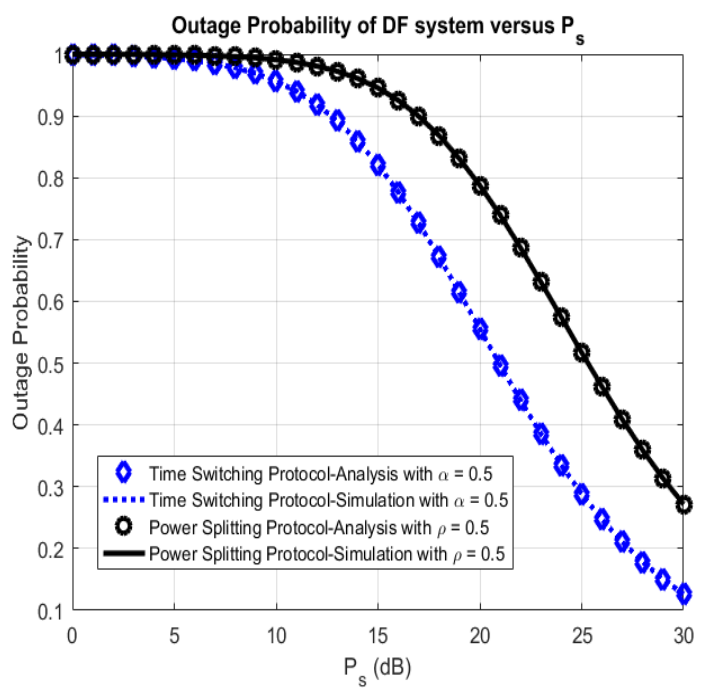

(b) Outage Probability versus $P_{s}$

Fig. 7: Delay-limited transmission.

\section{Conclusions}

In this paper, the system performance of the DF relaying network in the delay-limited and the delay-tolerant transmission modes have been proposed and analyzed. In this model, both the time switching and the power splitting protocols are fully considered. From that system model, the throughput, outage probability and the ergodic capacity of a DF relaying network 
are demonstrated by analytical mathematical algorithm and the Monte Carlo simulation. The research results show that the simulation and the analytical system performance are agreed with each other. The numerical analysis in this paper has provided practical approach into the effect of the interference noise on the system performance of wireless energy harvesting and information processing with DF relay nodes

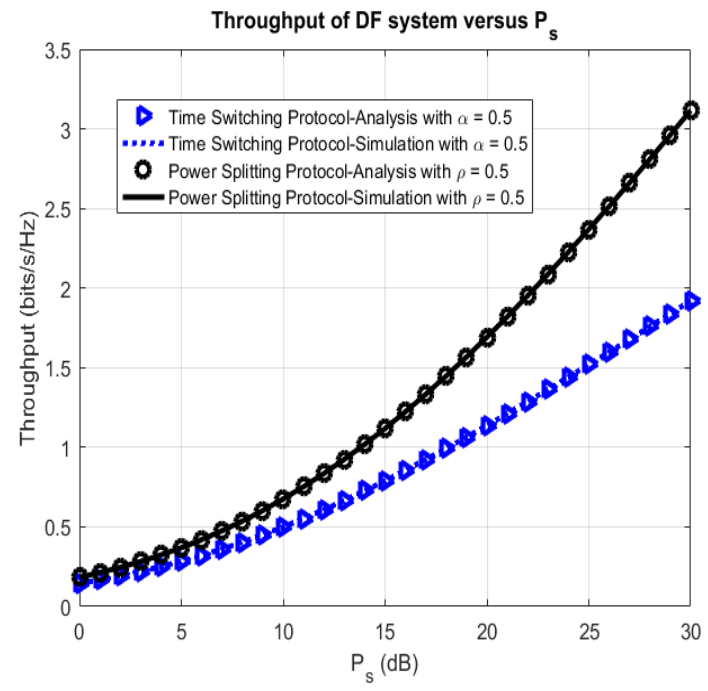

(a) Throughput versus $P_{s}$

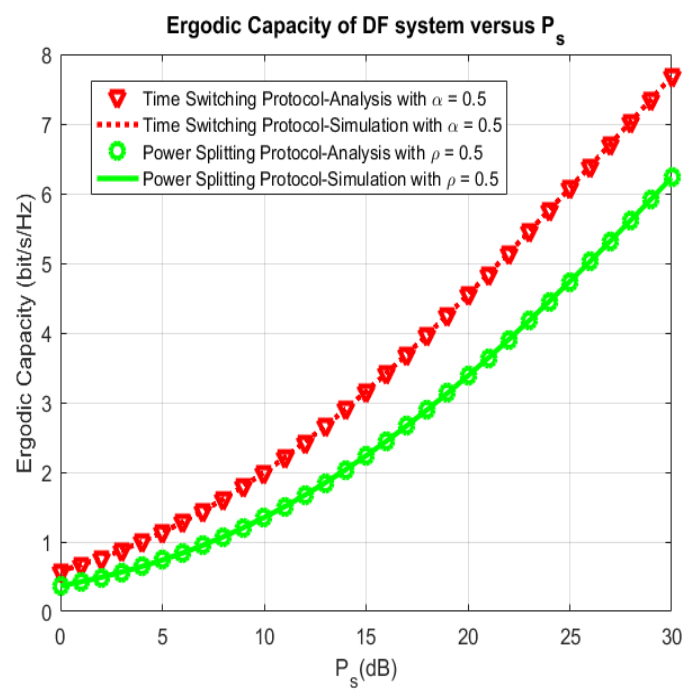

(b) Ergodic Capacity versus $P_{s}$

Fig. 8: Delay-Tolerant transmission.

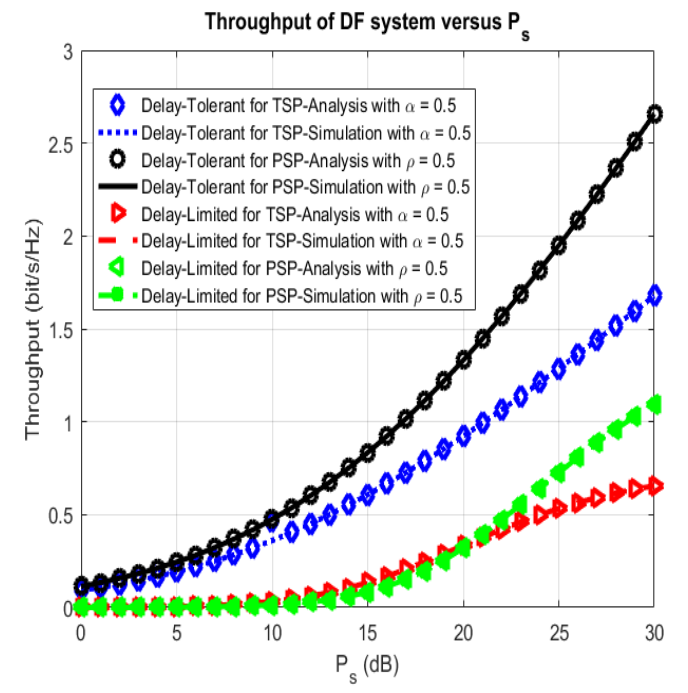

Fig. 9: Throughput of DF system versus $P_{s}$.

\section{ACKNOWLEDGEMENT}

The authors appreciate the support of Dr. Phuong T. Tran Vice-Dean at Faculty of Electrical and Electronics Engineering, Ton Duc Thang University, Ho Chi Minh City, Vietnam.

\section{References}

[1] NIYATO, D., D. I. KIM, M. MASO, Z. HAN, "Wireless Powered Communication Networks: Research Directions and Technological Approaches", IEEE Wireless Communications, 2-11, 2017.

[2] ANNUNZIATO, A., "5G Vision: NGMN 5G Initiative", 2015 IEEE 81st Vehicular Technology Conference (VTC Spring), vol. 5, 2015.

[3] LU, X., D. NIYATO, P. WANG, D. I. KIM, "Wireless charger networking for mobile devices: Fundamentals, standards, and applications," IEEE Wireless Communications, vol. 22, no. 2, pp. 126-135, 2015.

[4] LIU, J., H. GUO, H. NISHIYAMA, H. UJIKAWA, K. SUZUKI, N. KATO, "New Perspectives on Future Smart FiWi Networks: Scalability, Reliability, and Energy Efficiency," IEEE Communications Surveys 
and Tutorials, vol. 18, no. 2, pp. 1045-1072, 2017.

[5] RAMEZANI, P., A. JAMALIPOUR, "Throughput Maximization in Dual-Hop Wireless Powered Communication Networks," IEEE Transactions on Vehicular Technology, 1-1, 2017

[6] NGUYEN, T. N., T. T. DUY, G. LUU, P. T. TRAN, M. VOZNAK, "Energy Harvesting-based Spectrum Access with Incremental Cooperation, Relay Selection and Hardware Noises," Radioengineering, vol. 26, no. 1, pp. 240-250, 2017.

[7] NASIR, A. A., X. ZHOU, S. DURRANI, R. A. KENNEDY, "Relaying Protocols for Wireless Energy Harvesting and Information Processing," IEEE Transactions on Wireless Communications, vol. 12, no. 7, pp. 3622-3636, 2013.

[8] HUANG, K., E. LARSSON, "Simultaneous Information and Power Transfer for Broadband Wireless Systems," IEEE Transactions on Signal Processing, vol. 61, no. 23, pp. 5972-5986, 2013.

[9] BI, S., C. K. HO, R. ZHANG, "Wireless powered communication: Opportunities and challenges," IEEE Communications Magazine, vol. 53, no. 4, pp. 117-125, 2015 .

[10] ZHANG, Y., J. XUE, T. RATNARAJAH, "Capacity analysis for multi-antenna dualhop AF system with random co-channel interference," IET Communications, vol. 11, no. 9, pp. $1454-1465,2015$.

[11] HUANG, Y., F. AL-QAHTANI, C. ZHONG, Q. WU, J. WANG, H. ALNUWEIRI, "Performance Analysis of Multiuser Multiple Antenna Relaying Networks with Co-Channel Interference and Feedback Delay," IEEE Transactions on Communications, vol. 62, no. 1, pp. 59-73, 2014.

[12] NGUYEN, T. N., D. T. DO, P. T. TRAN, M. VOZNAK, "Time Switching for Wireless Communications with Full-Duplex Relaying in Imperfect CSI Condition," KSII
Transactions on Internet and Information Systems, vol. 10, no. 9, 2016.

[13] LIU, L., R. ZHANG, K. CHUA, "Wireless Information Transfer with Opportunistic Energy Harvesting," IEEE Transactions on Wireless Communications, vol. 12, no. 1, pp. 288-300, 2013.

[14] LIU, L., R. ZHANG, K. CHUA, "Wireless Information and Power Transfer: A Dynamic Power Splitting Approach," IEEE Transactions on Communications, vol. 61, no. 9, pp. 3990-4001, 2013.

[15] ZHANG, R., C. K. HO, "MIMO Broadcasting for Simultaneous Wireless Information and Power Transfer," IEEE Transactions on Wireless Communications, vol. 12, no. 5, pp. 1989-2001, 2013.

[16] PARK, J., B. CLERCKX, "Joint Wireless Information and Energy Transfer in a TwoUser MIMO Interference Channel," IEEE Transactions on Wireless Communications, vol. 12, no. 8, pp. 4210-4221, 2013.

[17] CHALISE, B. K., Y. D. ZHANG, M. G. AMIN, "Energy harvesting in an OSTBC based amplify-and-forward MIMO relay system," 2012 IEEE International Conference on Acoustics, Speech and Signal Processing (ICASSP), 2012.

[18] TUTUnCUOGLU, K., A. YENER, "Cooperative energy harvesting communications with relaying and energy sharing," 2013 IEEE Information Theory Workshop (ITW)., 2013.

[19] KRIKIDIS, I., S. TIMOTHEOU, S. SASAKI, "RF Energy Transfer for Cooperative Networks: Data Relaying or Energy Harvesting," IEEE Communications Letters, vol. 16, no. 11, pp. 1772-1775, 2012.

[20] NISHIMOTO, H., Y. KAWAHARA, T. ASAMI, "A prototype implementation of ambient RF energy harvesting wireless sensor networks," IEEE Sensors, vol. 11, 2011.

[21] Table of Integrals, Series, and Products. (2015). 


\section{About Authors}

Miroslav VOZNAK (born in 1971) is an Associate Professor in the Department of Telecommunications, Technical University of Ostrava, Czech Republic and foreign professor with Ton Duc Thang University in Ho Chi Minh City, Vietnam. He received his Ph.D. degree in telecommunications in 2002 at the Technical University of Ostrava. He is a senior researcher in the Supercomputing center IT4Innovations in Ostrava, Czech Republic, a member of the Scientific Board of FEI VSB-TU Ostrava, editorial boards of several journals and boards of international conferences. Topics of his research interests are IP telephony, wireless networks, speech quality and network security.

Hoang Quang Minh TRAN received his Ph.D. from Tomsk Polytechnic University, Tomsk City, Russian Federation. His research interests include high-voltage power systems,

"This is an Open Access article distributed under the terms of the Creative Commons Attribution License, which permits unrestricted use, distribution, and reproduction in any medium, provided the original work is properly cited (CC BY 4.0)." optoelectronics, wireless communications and network information theory. He serves as Lecturer in the Faculty of Electrical and Electronics Engineering, Ton Duc Thang University, Ho Chi Minh City, Vietnam.

Nhat Tan NGUYEN was born in 1986 in Nha Trang City, Vietnam. He received B.S. and M.S. degrees in Electronics and Telecommunications Engineering from $\mathrm{Ho}$ Chi Minh University of Natural Sciences, a member of Vietnam National University at Ho Chi Minh City (Vietnam) in 2008 and 2012, respectively. In 2013, he joined the Faculty of Electrical and Electronics Engineering of Ton Duc Thang University, Vietnam as a lecturer. He is currently pursuing his Ph.D. degree in Electrical Engineering at VSB Technical University of Ostrava, Czech Republic. His major interests are cooperative communications, cognitive radio, and physical layer security. 